\title{
An Ad Hoc Mobility Model Founded on Social Network Theory
}

\author{
Mirco Musolesi \\ Dept. of Computer Science, \\ University College London \\ Gower Street, London \\ WC1E 6BT, United Kingdom \\ m.musolesi@cs.ucl.ac.uk
}

\author{
Stephen Hailes \\ Dept. of Computer Science, \\ University College London \\ Gower Street, London \\ WC1E 6BT, United Kingdom \\ s.hailes@cs.ucl.ac.uk
}

\author{
Cecilia Mascolo \\ Dept. of Computer Science, \\ University College London \\ Gower Street, London \\ WC1E 6BT, United Kingdom \\ c.mascolo@cs.ucl.ac.uk
}

\begin{abstract}
Almost all work on mobile ad hoc networks relies on simulations, which, in turn, rely on realistic movement models for their credibility. Since there is a total absence of realistic data in the public domain, synthetic models for movement pattern generation must be used and the most widely used models are currently very simplistic, the focus being ease of implementation rather than soundness of foundation. Whilst it would be preferable to have models that better reflect the movement of real users, it is currently impossible to validate any movement model against real data. However, it is lazy to conclude from this that all models are equally likely to be invalid so any will do.

We note that movement is strongly affected by the needs of humans to socialise in one form or another. Fortunately, humans are known to associate in particular ways that can be mathematically modelled, and that are likely to bias their movement patterns. Thus, we propose a new mobility model that is founded on social network theory, because this has empirically been shown to be useful as a means of describing human relationships. In particular, the model allows collections of hosts to be grouped together in a way that is based on social relationships among the individuals. This grouping is only then mapped to a topographical space, with topography biased by the strength of social ties.

We discuss the implementation of this mobility model and we evaluate emergent properties of the generated networks. In particular, we show that grouping mechanism strongly influences the probability distribution of the average degree (i.e., the average number of neighbours of a host) in the simulated network.
\end{abstract}

\section{Categories and Subject Descriptors}

C.4 [Performance of Systems]: Modelling techniques; C.2.1 [Network Architecture and Design]: Wireless Communication

Permission to make digital or hard copies of all or part of this work for personal or classroom use is granted without fee provided that copies are not made or distributed for profit or commercial advantage and that copies bear this notice and the full citation on the first page. To copy otherwise, to republish, to post on servers or to redistribute to lists, requires prior specific permission and/or a fee.

MSWiM'04, October 4-6, 2004, Venezia, Italy.

Copyright 2004 ACM 1-58113-953-5/04/0010 ...\$5.00.

\section{General Terms}

Experimentation, Design

\section{Keywords}

Mobility model, mobile ad hoc networking, social networks

\section{INTRODUCTION}

The definition of realistic mobility models is one of the most critical and, at the same time, difficult aspects of the simulations of applications and systems designed for mobile environments. Currently, there is no publicly available data capturing node movement in real large-scale mobile ad hoc environments. Instead, synthetic models are used, and many such models have been presented in recent years [3]. The most widely used such models are based around random individual movement; the simplest, the Random Walk Mobility Model (equivalent to Brownian motion), is used to represent pure random movements of the entities of a system [4]. A slight enhancement of this is the Random Way-Point Mobility Model, in which pauses are introduced between changes in direction or speed.

All synthetic movement models are suspect because there is no means of assessing to what extent they map reality. However, it is not difficult to see by eye that the random mobility models generate behaviour that is most unhumanlike. This last point is key. Mobile devices are usually carried by humans, so the movement of such devices is necessarily based on human decisions and socialisation behaviour. Thus, for example, it is important to model the behaviour of individuals moving in groups and between groups, as is likely in the typical ad hoc networking deployment scenarios of disaster relief teams, platoons of soldiers, etc. In order to capture this type of behaviour, it is necessary to define models for group mobility that are heavily dependent on the structure of the relationships among the people carrying the devices. Existing group mobility models fail to capture this social dimension.

Taken together, for those systems in which mobility is important and for which a synthetic mobility model is an essential ingredient, it would appear to be important to consider the influence of the human-level social network as something that informs likely individual and group mobility patterns. Fortunately, in recent years, such networks have been investigated in considerable detail, both in sociology and in other areas, most notably mathematics and physics. Excellent re- 
views of the recent progress in complex and social networks analysis may be found in [1] and [11]. Mathematical models of such networks have been empirically shown to be useful in describing many types of relationships, including real social relationships [14].

In this paper, we propose a new two-level mobility model that is founded on artificially generated social relationships among the individuals carrying the mobile devices. The generation process respects the mathematical basis of social networks theory and is therefore grounded in empirical experience of actual social relationships. As part of this, we consider both individuals and groupings of individuals as first class entities in the model. The second stage of the model maps the social organisation onto topographical space in such a way that the actual topography generated is biased by the strength of social ties that, in reality, determine the likelihood of colocation.

The model we propose is relatively simple, but is parameterisable in a way that allows different scenarios to be modelled both at the level of social organisation and topographical translation. Only a single alternative mobility model founded on the relationships between people has been presented [6]; in this, the composition of the groups is static and it lacks a rigorous mathematical representation of the relationships between the individuals. Both points are addressed within this paper.

The remainder of this paper is organised as follows. Section 2 describes the design of our mobility model in detail. The implementation of the model is presented in Section 3, together with an analysis of its possible applications; some rather interesting statistical simulation results of the emergent properties of the model are also presented. Section 4 contains a critical analysis of our solution and a comparison to other models and research in this space. Finally, Section 5 concludes the paper, outlining our future research directions.

\section{DESIGN OF THE MOBILITY MODEL}

\subsection{Modelling Social Relationships}

Recent results in social network theory can be used to design more realistic mobility models for mobile ad hoc research. In particular, we represent a social network using a weighted graph, by defining the weights associated with each edge of the network to model the strength of the direct interactions between individuals. In this case, interactions are said to be direct if they take place between people who are colocated. It is our explicit assumption that these weights, which are expressed as a measure of the strength of social ties, can also be read as a measure of the likelihood of geographic colocation, though the relationship between these quantities is not necessarily a simple one, as will become apparent.

We model the degree of social interaction between two people using a value in the range $[0,1]$. 0 indicates no interaction; 1 indicates a strong social interaction. It is worth noting that these indicators are not a measure of the subjective importance of the relationships, such as family ties or friendships. Let us consider the case of a person working in a town that is different from the one in which his parents live. In this case, the social relationship is strong from a genealogical (and affective) point of view, but is weak if we consider the likelihood of direct interaction between them.
In other words, in our model, this relationship will be modelled using a low value (but not as low as between the person and an unconnected individual in their parents' town). An example of strong social interaction may be the case of two colleagues sharing the same office.

We use a matrix $\mathbf{M}$, which we call Interaction Matrix, to store this information. One example may be the following:

$\mathbf{M}=\left[\begin{array}{ccccccccc}1 & 0.75 & 0.60 & 0.91 & 0.11 & 0.05 & 0.00 & 0.03 & 0.20 \\ 0.75 & 1 & 0.23 & 0.81 & 0.24 & 0.03 & 0.13 & 0.18 & 0.21 \\ 0.60 & 0.23 & 1 & 0.30 & 0.28 & 0.03 & 0.01 & 0.02 & 0.17 \\ 0.91 & 0.81 & 0.30 & 1 & 0.65 & 0.13 & 0.14 & 0.23 & 0.04 \\ 0.11 & 0.24 & 0.28 & 0.65 & 1 & 0.23 & 0.13 & 0.11 & 0.05 \\ 0.05 & 0.03 & 0.03 & 0.13 & 0.23 & 1 & 0.83 & 0.44 & 0.55 \\ 0.00 & 0.13 & 0.01 & 0.14 & 0.13 & 0.83 & 1 & 0.71 & 0.03 \\ 0.03 & 0.18 & 0.02 & 0.23 & 0.11 & 0.44 & 0.71 & 1 & 0.94 \\ 0.20 & 0.21 & 0.17 & 0.04 & 0.05 & 0.55 & 0.03 & 0.94 & 1\end{array}\right]$

The generic element $m_{i, j}$ represents the interaction between two individuals $i$ and $j$. We refer to the elements of the matrix as the interaction indicators. The diagonal elements represent the relationships that an individual has with himself and are set, conventionally, to 1 . If the interaction indicator between two individuals $i$ and $j$ is less than 0.25 , they are considered socially disconnected. The choice of the value 0.25 is arbitrary and it is only used to provide a clearer graphical representation of the important connections between people.

The matrix is symmetric since, to a first approximation, interactions can be viewed as being symmetric. It is, however, worth underlining the fact that we are using a specific measure of the strength of the relationships. Using psychological tests it is probable that the importance of a relationship, such as a friendship, will be valued differently by the different individuals involved; in our modelisation, this would lead to an asymmetric matrix. We plan to investigate this issue further in the future.

Each individual is also characterised by an Sociability Factor (SF), that is an indicator of its attitude towards interaction with others. The Sociability Factor of an individual $i$ is obtained using the following formula:

$$
S F_{i}=\frac{\sum_{\substack{j=1 \\ j \neq i \\ m_{i, j}>c t}}^{n} m_{i, j}}{z}
$$

where $c t$ is the connection threshold (in our case 0.25). In other words, we consider only the people who have a significant social relationship with person $i$. $z$ is the total number of people in the system characterised by $m_{i, j}>c t$ (i.e., the number of neighbours in the social network). A sociable host will have a SF close to 1 and a solitary one will be then characterised by a SF close to 1 .

\subsection{Establishment of the Social Model}

For reasons of clarity, we make the assumption that only one device is associated with each individual. However, again, the model can easily be extended. In the remainder of the discussion, we note that the terms host, node and individual are equivalent and indicate a single moving entity in the mobility scenario.

Our aim is to use the social relationships among individuals to define groups of hosts that move together in the simulated scenarios. Moreover, we use this social network as a basis for the dynamics of the simulated environment. 
The first step in this two-level process is the generation of the social network; that is, the generation of the Interaction Matrix, using random distributions. It is possible to choose different distributions according to the specific modelling requirements. For example, it is possible to choose a uniform distribution for the generation of equiprobable interaction indicators or a Poisson distribution to model a scenario where the connections are characterised by interaction indicators that are denser around a given value. Another development in which we are currently engaged stems from the creation of a possible variant of the Albert-Barabasi model [1] to generate weighted scale-free social networks. Using the generated interaction indicators, the Sociability Factors are calculated for each host as described in Section 2.1 .

Grouping can occur at two levels. Firstly, grouping naturally arises at the social level, and connected social groups are defined in the process of creating the interaction matrix by manipulating the interaction indicators. However, social groupings only indicate a propensity to be colocated; in a geographical sense, individuals form groups more dynamically, by socialising with different subgroups of people with whom they are socially related at different times (e.g., office and home groups) and sometimes by not socialising with such groups at all. Thus geographic grouping is a different concept to social grouping and must be treated differently within the model. In the main, individuals will move within the sphere of influence of the geographic group with which they are associated at any given point in time, but, occasionally, they will decide either to move between groups or to leave the group structure and to move completely independently. This decision process is biased by both social attractiveness of different groups and by the difficulty of reaching each group, which we represent by a function of the distance to that group. In the current instantiation of the model, the number of geographic groups to which an individual may belong, and the radius of such groups, are parameters of the model and are statically defined (we refer to the number of geographical groups with $n_{\text {groups }}$ ). This is something of an oversimplification, and we are currently engaged in identifying dynamic mechanisms that allow changes in the numbers of groups over time; in other words, geographic grouping will itself be an emergent property of the model in future.

Given the value of $n_{\text {groups }}$, the hosts are ordered using their SFs. Then, starting from the host with the highest SFs, the first $n_{\text {groups }}$ hosts are randomly assigned to different groups. After this, the remaining hosts are iteratively assigned to each group according to the intensity of the relationships between the host and the members that are present in the various groups. More specifically, the attraction intensity of a certain group $G$ towards a host $i$ is given by the sum of the interaction indicators that describes the relationships between the host $i$ and the nodes in group $G$. We call this quantity the group attraction towards a certain host. More formally, the attraction exerted by a group $G$ towards a host $i$ is calculated using the following formula

$$
G A_{i, G}=\frac{\sum_{\substack{j=1 \\ j \in G}}^{n} m_{i, j}}{f}
$$

where $f$ is the number of members in the group.

A possible refinement of the model derives from an em- pirical consideration. Generally, it is more probable that a person joins a group of friends/acquaintances if this is close to him/her. In order to model this phenomenon, we introduce the distance $r$ between the host and the group ${ }^{1}$ as denominator in the previous formula as follows

$$
G A_{i, G}=\frac{\sum_{\substack{j=1 \\ j \in G}}^{n} m_{i, j}}{f r^{k}}
$$

where $f$ is the number of the members of the group and $k$ is a parameter that can be tuned in accordance with the possible various modelling requirements. It is worth noting that, by setting $k=0$, the previous formula is obtained (i.e., the distance has no influence). Higher values of $k$ increases the influence of the distance in the computation of the Group Attraction values. The last $h$ hosts in the ordered list are located in the space between the group areas. This means that the initial instantiation of the social model in geographic form may have some hosts that do not initially belong to any group. A group (or cloud) area is associated with each group of hosts. Each group area is defined using a geometric shape. In our model, shapes can be square or circular. Assuming a Cartesian system, rectangular areas are defined by selecting the coordinates $(\min X, \min Y)$, which represent the point of the cloud with the minimum distance from the origin, and the length of the side. Similarly, circular areas are defined by selecting the centre of the circle and its radius.

\subsection{Position Selection and Update}

In this section, we will discuss the mechanisms that form the basis of the evolution of the simulated scenarios after the initial establishment phase.

A host belonging to a group moves inside the corresponding group area towards a goal (i.e, a point randomly chosen in the group space) using the standard Random Way-Point model. It is worth noting that clouds also move towards randomly chosen goals in the simulation space.

Each group moves with a random speed (with a value contained in a predefined range); moreover, each host moves with a randomly generated different speed (once again, contained in a predefined range). Therefore, the movement of a host that belongs to a cloud is the result of the composition of these speeds. The equations used to update the position of the host are the following:

$$
\begin{aligned}
& \text { new } X_{\text {node }_{i}}=\text { current }_{\text {node }_{i}} \pm \text { speed }_{\text {node }_{i}} \Delta t \pm \text { speed }_{\text {group }_{i}} \Delta t \\
& \text { new }_{\text {node }_{i}}=\text { current }_{\text {node }_{i}} \pm \text { speed }_{\text {node }_{i}} \Delta t \pm \text { speed }_{\text {group }} \Delta t
\end{aligned}
$$

We use the following simple equations for the position of each group:

$$
\begin{aligned}
& \text { new }_{\text {group }_{i}}=\text { current }_{\text {group }_{i}} \pm \text { speed }_{\text {group }_{i}} \Delta t \\
& \text { new }_{\text {group }_{i}}=\text { current }_{\text {group }_{i}} \pm \text { speed }_{\text {group }} \Delta t
\end{aligned}
$$

Similarly, the hosts that are not in cloud areas move towards goals that are randomly chosen in the simulation area. The positions of all the hosts and groups are updated every $\Delta t$ seconds

\footnotetext{
${ }^{1}$ More precisely, we consider the distance between the host and the geometrical centre of the group area.
} 


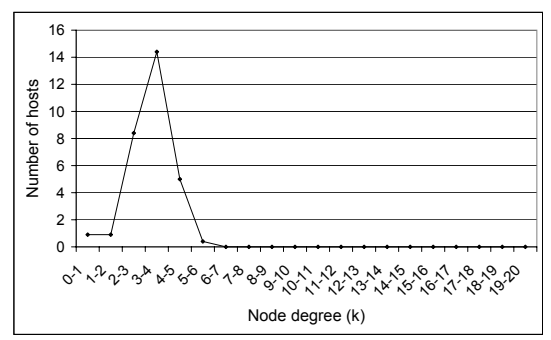

Figure 1: Distribution of degree of connectivity (scenario with 30 hosts grouped into 5 groups).

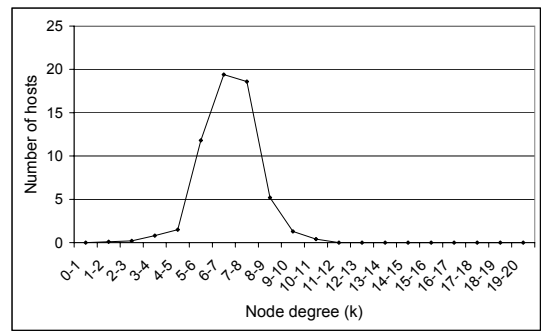

Figure 2: Distribution of degree of connectivity (scenario with 60 hosts grouped into 5 groups).

\subsection{Modelling Hosts and Groups Dynamics}

In the previous section, we presented a general overview of the model. In this section we describe how social network relationships influence the evolution and the dynamics of the simulated mobile scenario.

Let us consider the case of a host inside a group. When a host reaches a goal, it implicitly reaches a decision point at which it must decide whether to remain within the group, to move to another group, or to escape outside all groups. This process is driven by the Sociability Factor of the host. More specifically, a threshold is generated using a uniform random distribution; if the Sociability Factor of the host is higher than the threshold, a new goal is chosen outside the areas of any group. If this does not happen, a new goal inside one of the groups (including the current one) is chosen. More specifically, the attraction intensities exerted by the groups towards the host are calculated. The host will join the group that exerts the highest attraction. If the group, of which the host is currently a part exerts the greater attraction, the host will not leave the group.

The case of a host starting outside group areas is symmetric. When the host reaches its goal, a threshold is generated and if the Sociability Factor of the host is lower than the threshold, the host will join the group of hosts that exerts the greatest attraction.

\section{IMPLEMENTATION AND EVALUATION}

In order to extract quantitative information about the structure of the generated mobile scenario, the mobility model was implemented using OmNet++ [13], a discrete event simulator. We used uniform distributions to generate the Interaction Matrices and the connection threshold was set to 0.25

We considered two scenarios characterised by different numbers of hosts and groups. We defined a square simulation area with a side of $1 \mathrm{~km}$ and group areas with a side of $200 \mathrm{~m}$. The simulation was set to run for 1 hour of simulated time (10 replicates for each mobile scenario) in order to obtain a statistically meaningful set of results. Each group moves with a random speed (with a value in the range 1-2 $\mathrm{m} / \mathrm{s}$ ) and each host moves with a randomly generated different speed (with a value in the range $1-3 \mathrm{~m} / \mathrm{s}$ ). As described above, the movement of a host is the result of the composition of these speeds. The first scenario was composed of 30 hosts grouped into 5 geographically separate groups, whereas the second was composed of 60 hosts grouped into the same number of groups. In both cases, $80 \%$ of the hosts were initially placed inside the groups.

From the simulation results, we extracted the distribution of the average degree of connectivity. The average is computed using a sample interval equal to 1 second. Figure 1 and Figure 2 show the distributions of the degree of connectivity related to the scenarios composed of 30 and 60 hosts respectively, each with five groups. The resulting distributions of the degree of the networks are typically Poissonlike, as in mobile ad hoc networks in which the hosts have random movements [5]. However, the social clustering influences the dynamic network topology and, consequently, the average node degree, as it can be seen by comparing the range of values of $k$ corresponding to the "peak" of the function $(<k>)$ in Figures 1 and 2. The value of $\langle k\rangle$ in Figure 2 roughly doubles with respect to Figure 1 (from the range $3-4$ to the range 6-8), indicating that, approximately, double the number of the nodes are now clustered in the group areas ${ }^{2}$.

\section{DISCUSSION AND RELATED WORK}

In our previous research work about the definition of a context-aware adaptive routing protocol [9] and the design of an epidemic routing based messaging middleware [10] for ad hoc networks, we developed a group mobility model in which the composition of groups is based on probabilistic mechanisms. We also presented some solutions for the evolution of the simulated scenarios in terms of movement of the hosts between the various groups.

Many mobility models have been presented for the testing of protocols and algorithms of mobile ad hoc networks. A comprehensive review of the most popular mobility models used by the mobile ad hoc research community can be found in [3]. However, it is interesting and, at the same time, surprising to note that even the best solutions and approaches have only been tested using completely random models such as the Random Way-Point model, without grouping mechanisms, or using other simple groups mobility models, like [7]. The almost pervasive adoption of such models has generated a considerable amount of work that is predicated on the reasonableness of random mobility models.

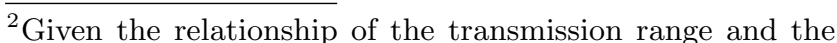
side length of the group areas (both equal to $200 \mathrm{~m}$ ), roughly all the hosts in a group are connected in our simulation. Therefore, $k$ is strictly correlated to the average number of hosts per group. 
The work most directly related to ours can be found in [6]. This work is based on similar assumptions to ours, but is considerably more limited in scope. For example, hosts are statically assigned to a particular group during the initial configuration process whereas our model accounts for movement between groups. Moreover, the authors claim that mobile ad hoc networks are scale-free, but the typical properties of scale-free networks are not exploited in the design of the model presented by the authors. The scale-free distribution of mobile ad hoc networks is still not proven in general, since practical measurements are not currently available. However, it is worth noting that the scale-free properties are strictly dependent on the movements of hosts and therefore are dependent on the actual simulated scenarios [5].

In the recent years, many researchers have tried to refine existing models in order to make them more realistic. For example, in [8], a technique for the creation of more realistic mobility models that include the presence of obstacles is presented. The specification of obstacles is based on the use of Voronoi graphs in order to derive the possible pathways in the simulation space. This approach is orthogonal to ours, and it is possible to modify our framework in order to include the presence of obstacles using similar techniques; only the equations used to update the positions of the entities need to be modified.

Some interesting studies have been recently carried out on the connectivity of ad hoc networks with respect to complex networks theory. For example, Glauche et al. in [5] discuss some network properties using percolation theory; that is, an application of complex networks theory derived by the investigation of physical phenomena such as phase transitions in molecular lattices. In [12], the authors presents mathematical results about the possible emergence of scalefree structures in ad hoc networks. However, the authors consider only fixed ad hoc networks (such as peer-to-peer networks), without analysing the influence of movement in the definition of their model.

\section{FUTURE WORK AND CONCLUSIONS}

Existing mobility models, on which much ad hoc networks research is founded, are based on highly simplistic random movement models. Since these models are patently unrealistic, the practical applicability of much current ad hoc networks research must be considered highly suspect.

In the absence of trace data, the best that can be achieved is to base synthetic mobility models on realistic models of human socialisation. In this paper, we have presented a novel group mobility model for mobile ad hoc networks research, founded on social network theory. Since mobility models can only be judged on the basis of behaviour that emerges as a consequence of their dynamic evolution, we have discussed the emergent properties of the networks generated using our model. We have shown, in particular, that the degree of the simulated network is strongly influenced by the grouping mechanisms.

We are now investigating other properties of networks generated by our model from a theoretical point of view. In particular, we intend to study the network topology using some remarkable mathematical results about random graphs presented in [2]. The mobility model presented in this paper will be used in our current investigation about the design of efficient routing protocols and systems (especially in terms of the use of the available resources) for mobile ad hoc net- works. We believe that it is possible to design mechanisms based on the evaluation of the social network that connects the individuals carrying the mobile devices, in order to build more efficient and, at the same time, more reliable systems. For example, in order to reduce the number of replicas that are presented in the system, it is possible to consider the likelihood of the colocation of certain hosts.

Finally, we plan to refine the model both by making dynamic changes in the number of groups and by allowing the definition of obstacles within the simulation environment.

\section{REFERENCES}

[1] R. Albert and A.-L. Barabasi. Statistical mechanics of complex networks. Review of Modern Physics, 74:47-97, 2002

[2] B. Bollobas. Random Graphs. Cambridge University Press, Second edition, 2001.

[3] T. Camp, J. Boleng, and V. Davies. A survey of mobility models for ad hoc network research. Wireless Communication and Mobile Computing Special Issue on Mobile Ad Hoc Networking: Research, Trends and Applications, 2(5):483-502, 2002.

[4] A. Einstein. Investigations on the Theory of the Brownian Movement. Dover Publications, 1956.

[5] I. Glauche, W. Krause, R. Sollacher, and M. Greiner. Continuum percolation of wireless ad hoc communication networks. Phisica A, 325:577-600, 2003.

[6] K. Hermann. Modeling the sociological aspect of mobility in ad hoc networks. In Proceedings of MSWiM'03, San Diego, California, USA, September 2003.

[7] X. Hong, M. Gerla, G. Pei, and C.-C. Chiang. A group mobility model for ad hoc networks. In Proceedings of the 2nd International Workshop on Modeling Analysis and Simulation of Wireless and Mobile Systems, pages 53-60, 1999.

[8] A. Jardosh, E. M. Belding-Royer, K. C. Almeroth, and S. Suri. Towards realistic mobility models for mobile ad hoc networks. In Proceedings of MobiCom'03, San Diego, California, USA, September 2003.

[9] M. Musolesi, S. Hailes, and C. Mascolo. Adaptive routing for intermittently connected mobile ad hoc networks. Technical report, UCL-CS Research Note, July 2004. Submitted for Publication.

[10] M. Musolesi, C. Mascolo, and S. Hailes. Adapting asynchronous messaging middleware to ad hoc networking. In Proceedings of 2nd International Workshop on Middleware for Pervasive and Ad-Hoc Computing (co-located with Middleware 2004), Toronto, Canada, October 2004. ACM Press.

[11] M. E. J. Newman. The structure and function of complex networks. SIAM Review, 19(1):1-42, 2003

[12] N. Sarshar and R. Chowdhury. Scale-free and stable structures in complex ad hoc networks. Physical Review E 026101, 2003.

[13] A. Vargas. The OMNeT++ discrete event simulation system. In Proceedings of the European Simulation Multiconference (ESM'2001), Prague, June 2001.

[14] D. J. Watts. Small Worlds The Dynamics of Networks between Order and Randomness. Princeton Studies on Complexity. Princeton University Press, 1999. 